\title{
Duisburger Kongress Mathematik in Industrie und Wirtschaft 10.-13. März 1997
}

\section{von Georg Bock}

Mindestens in ihren anwendungsträchtigen Bereichen hat sich in den letzten Jahren das Bild der Mathematik so schnell gewandelt wie in kaum einer anderen Disziplin. Der wichtigen Grundlagenforschung stehen immer mehr komplexe, wissenschaftlich anspruchsvolle Anwendungen der Mathematik zur Seite, die in Industrie und Wirtschaft benötigt werden und die die Mathematik in die Rolle einer Schlüsseltechnologie versetzen. Da komplexe technische und wirtschaftliche Zusammenhänge nur mit ihr darstellbar und behandelbar sind, gewinnt die Mathematik eine Schlüsselrolle in allen Bereichen der „high technology“. Der universalen Wissenschaft Mathematik und den in ihr Ausgebildeten kommt dabei in zumeist interdisziplinären Kooperationen eine neue und zentrale Aufgabe bei der Lösung dringender praktischer Probleme zu. Mathematische Methoden haben durchschlagende Erfolge, erweisen sich immer mehr als wesentlicher Innovationsfaktor und schließlich als bedeutende ökonomische Ressource. Ihre unbestreitbaren Erfolge wecken allerdings auch hohe Erwartungen an die Mathematik.

Der Duisburger Kongress „Mathematik in Industrie und Wirtschaft" will diese Entwicklung fördern und dieses - für manche ungewohnte - aktuelle Bild der Mathematik der Öffentlichkeit vermitteln.

In sechs jeweils eintägigen Sektionen (von Dienstag bis Donnerstag)

- Steuerung und Simulation von Prozessen

- Kommunikation und neue Medien

- Umwelttechnologie und Energietechnik

- Risikobewertung in Banken und Versicherungen

- Werkstofftechnik und Konstruktion

- Logistik und Verkehrstechnik

werden mathematische Anwendungen aus Industrie und Wirtschaft dargestellt und neue mathematische Forschungsergebnisse für die Probleme der Praxis vorgestellt. „Success stories“ werden berichtet ebenso wie offene Probleme, von denen eine Lösung durch die Mathematik erwartet wird. Mehr als die Hälfte der Beiträge kommen dabei direkt aus der Praxis der Industrie und Wirtschaft.

Der Mittwoch ist zusätzlich einer speziellen Tagesveranstaltung für Lehrer, Schüler und Berufsberater gewidmet, die einen Einblick in das veränderte Bild der Hochschulmathematik und der mathematischen Berufspraxis gewinnen wollen. Eine Podiumsdiskussion mit Teilnehmern aus Politik, Industrie und Hochschulen über die Rolle der Mathematik als treibende, ja sogar dominierende Kraft in vielen technologischen Innovationen schließt den Tag ab.

Die Tagung wird begleitet von einem bunten Spektrum von Workshops, auf denen interessante Neuentwicklungen aus der Mathematik für die Anwendungen in Industrie und Wirtschaft vorgestellt werden (ab Montagmittag). Ausstellungen und Stände von mathematischen Zentren und Organisationen und Unternehmen der Industrie und Wirtschaft informieren über aktuelle Projekte. Posterstände geben Auskunft über neue mathematische Probleme und neue Lösungen für die Industriepraxis.

Wer die Duisburger Jahrestagung der DMV aus dem Jahre 1995 miterlebt hat, weiß im übrigen, daß die Kolleginnen und Kollegen am Ort auch einen anregenden Rahmen zu organisieren verstehen. Und das ist schließlich auch ein wichtiger Zweck der Veranstaltung: Mathematiktreibende aus Industrie und Wirtschaft, aus Hochschulen und Forschung, Politik und Verwaltung in interessanten Diskussionen zusammenzubringen und damit das Netzwerk von Ideen, Personen, Institutionen, Themen und Methoden enger zu knüpfen, auf das die Mathematik - nicht nur in Industrie und Wirtschaft - angewiesen ist.

Der Duisburger Kongress wird gemeinsam veranstaltet vom Arbeitskreis "Mathematik in Forschung und Praxis“ und der DMV-Fachgruppe „Industrieund Wirtschaftsmathematik" in Zusammenarbeit mit ECMI, SIAM und dem Fachbereich Mathematik der Universität Duisburg. Er wird gefördert von DFG, BMBF, Ministerium für Wissenschaft und Forschung NRW und zahlreichen Industriefirmen.

Anmeldungen und Informationen:

AK Mathematik in Forschung Praxis

FB 11, Universität Duisburg

Lotharstr. 65, 47048 Duisburg

kongress@math . uni-duisburg.de

http://www . uni-duisburg.de

/FB11/EVENTS/MiIW.html

Tel. 0203/379-3502

Fax 0203/379-3139

Adresse des Autors:

Prof. Dr. H. G. Bock

IWR

Universität Heidelberg

Im Neuenheimer Feld 368

69120 Heidelberg 